\title{
The Multiple Facets of Per2
}

\author{
U. Albrecht, A. Bordon, I. Schmutz, AND J. RIPPERGeR \\ Department of Medicine, Division of Biochemistry, University of Fribourg, 1700 Fribourg, Switzerland
}

\begin{abstract}
The Period 2 (Per2) gene is an important component of the circadian system. It appears to be not only part of the core oscillator mechanism, but also part of the input and output pathways of the clock. Because of its involvement at multiple levels of the circadian system, Per2 needs to meet a variety of different demands. We discuss how Per 2 might be able to fulfill multiple functions by reviewing known facts and combine this with speculations based on these facts. This might provide new views about Per 2 function and help to better understand diseases that are rooted in the circadian system.
\end{abstract}

\section{INTRODUCTION}

Life on earth has used the sun as a reference point to time biological processes over the 24 hours of a day. To optimize energy expenditure and uptake, a mechanism developed to predict the cyclic availability of light. Because the period length of one cycle of such a timing device is circadian (circa diem $=$ about 1 day) and not exactly 24 hours, it was termed a circadian clock. Due to its slight imprecision, the clock must be reset periodically to serve as a reliable predictor of solar time. Continuous clock adaptation is also needed because the earth's orbit around the sun generates the seasons that manifest themselves in alterations of day length over the year. To adapt to these changes, the circadian clock is connected to mechanisms that allow it to stay in tune with nature. Sensory organs communicate environmental time information via signaling pathways to the clock, thereby synchronizing the internal circadian oscillators with the environment. Therefore, the circadian clock can be viewed as the link between the environment and the genetic and biochemical machinery of an organism.

At the core of the mammalian molecular clock are two transcriptional activators (Clock and Bmall) and factors negatively acting on them (Per and $\mathrm{Cry}$ ). The heterodimer of Clock/Bmall binds to E-box elements present in the $P e r$ and $C r y$ promoters leading to their expression. The proteins enter the nucleus and disrupt Clock/Bmall action thereby suspending the transcription of their own genes and of the Rev-erb $\alpha$ gene. This gene codes for an orphan nuclear receptor that negatively regulates Bmall gene expression (Wijnen and Young 2006).

Because Per genes are at the core of the circadian clock mechanism, it is of interest to understand the function of these genes and proteins at the molecular level. Alterations in Per gene function affect many biochemical processes such as the cell cycle and metabolism. These in turn are linked to physiological process such as aging, brain dysfunction, and development of cancer. Hence, understanding Per function will unravel solutions to influence the aging process and provide insights into the treatment of cancer, depression, and metabolic diseases. In this chapter, we focus on the Per 2 gene in particular, because its malfunction has widespread effects on the organism, but its molecular function is largely unknown.

\section{GENERAL FEATURES OF PER2}

The Per 2 gene has been identified searching the Genbank database using human Perl (Rigui) for comparison (Albrecht et al. 1997). An open reading frame encoded by a human cDNA designated as KIAA0347 (Nagase et al. 1997) had high sequence homology with Perl and was designated Per2 (Albrecht et al. 1997; Shearman et al. 1997). Human Per2 was mapped to chromosome 2q37.3 (Toh et al. 2001). This gene was also found on chromosome 2 in zebra fish, opossum, and the chimpanzee (2b), whereas it is localized on chromosome 1 in the mouse, on chromosome 9 in the rat, on chromosome 12 in the macaque, and on scaffold 55 in Xenopus tropicalis. Homologs of Per2 have also been identified in birds, lizards, mole rats, horse, vole, and sheep, but their chromosomal localization has not been determined. Here, we only consider the human and mouse Per 2 genes, both containing 23 exons, coding for transcripts of $6220 \mathrm{bp}$ or $5805 \mathrm{bp}$ and proteins of 1255 amino acids or 1257 amino acids, respectively. It appears that the mammalian $\mathrm{Per}$ gene paralogs probably emerged through gene duplications from an ancestral gene, resulting in four paralogs of which Per4 was lost (von Schantz et al. 2006). Interestingly, mouse Per2 appears to be more similar to Drosophila per than to mouse Perl (Albrecht et al. 1997) or Per3.

\section{REGULATION OF PER2 GENE EXPRESSION}

The Per 2 gene is expressed in a circadian manner in the suprachiasmatic nucleus (SCN), and its expression is delayed by 4 hours as compared to Perl (Albrecht et al. 1997). Per 2 gene expression is not restricted to the brain but is expressed in almost all tissues. Furthermore, Per2 expression in the SCN of mice can be strongly induced by light in the early subjective night but not in the late subjective night in contrast to Perl (Fig. 1a,b) (Yan and Silver 2002). Interestingly, Per2 is also induced by blue light in the early subjective night in oral mucosa of humans (Cajochen et al. 2006). Apart from light, Per 2 can also be regulated by nonphotic stimuli such as 5-HT1A/7 receptor agonists (Horikawa et al. 2000), neuropeptide Y (Fukuhara et al. 2001), vasoactive intestinal polypeptide (Nielsen et al. 2002), glucocorticoids (Segall et al. 2006), and ghrelin (Yannielli et al. 2007). 
a

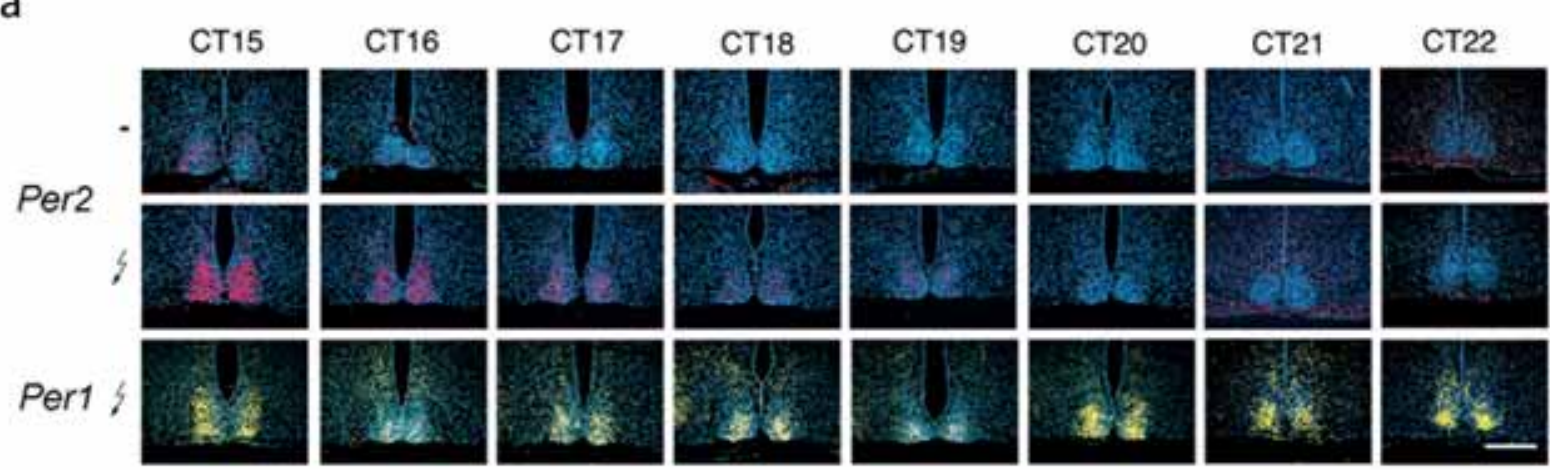

b
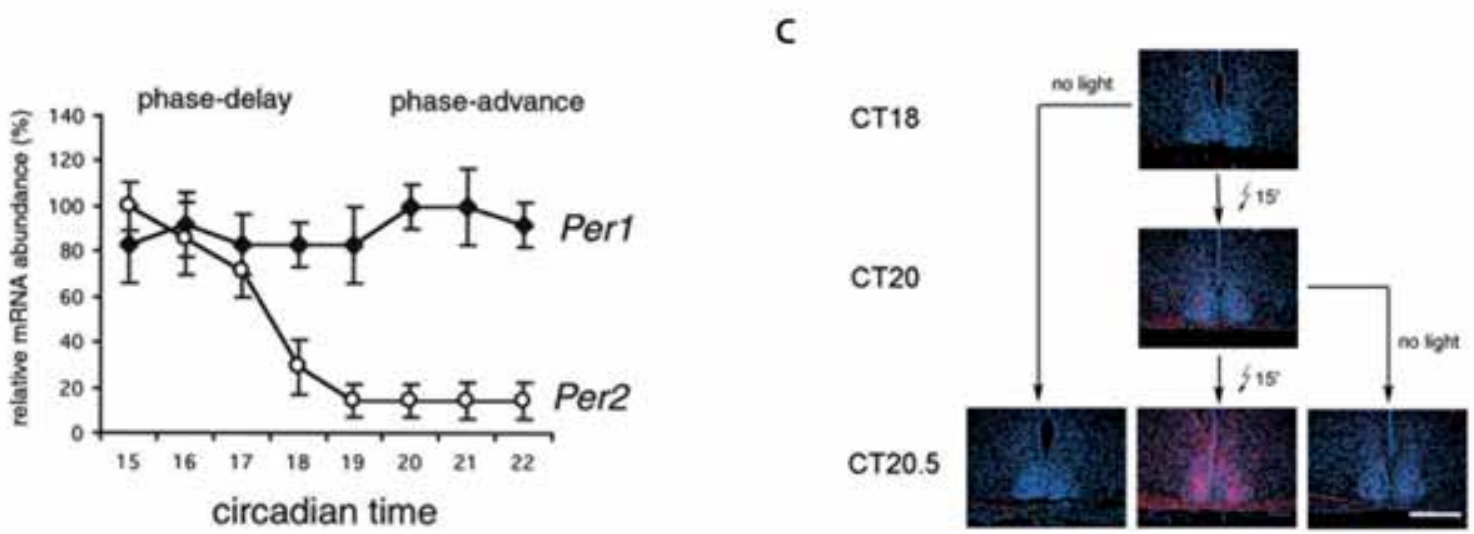

Figure 1. Light-induced levels of Per2 correlate with the behavioral phase-delay domain. (a) Induction of Per 1 (yellow) and Per2 (red) by a 15 -minute light pulse applied at the circadian times (CTs) indicated (blue = cell nuclei stained with Hoechst dye). Endogenous Per2 expression in control mice is low except for CT15, where residual expression is still detectable (top row). A light pulse induces this gene in a CT-dependent fashion (center row). Endogenous Perl expression is not expressed at any of the CTs examined, but it is induced by a light pulse in a CT-independent fashion (bottom row). Bar, $500 \mu \mathrm{m}$. (b) Graphical representation of the relationship between Perl and Per 2 induction and phase delay evoked by a light pulse. The abscissa plots the circadian time and the ordinate depicts the relative mRNA abundance with the maximal value for Perl set to $100 \%$. (Closed circles) Perl expression levels in the suprachiasmatic nucleus (SCN); (open circles) Per2 expression levels in the SCN. In the case of Per2, gene induction is correlated with the domain in the subjective night in which animals respond with a phase-delay to a light pulse. Each time point shows the mean \pm S.E.M. of three different animals. Perl and Per2 induction was assessed at each CT on adjacent sections of the same animal. (c) Effect of a double-light pulse at CT18 on Per2 expression in the SCN. A single 15-minute light pulse applied at CT18 induced Per 2 weakly (see also $a$ ). However, a second 15 -minute pulse evokes strong Per 2 expression. The control SCN that had not been exposed to light does not exhibit Per2 expression. Bar, $500 \mu \mathrm{m}$.

These characteristics indicate an intricate manner of transcriptional regulation of the Per 2 gene. Toward a system-level understanding of the transcriptional circuitry regulating circadian clocks, Ueda et al. (2005) identified a number of clock-controlled elements in the Per 2 promoter. A noncanonical E-box enhancer (CATGTG, -497 in human and -163 in mouse, and CACGTT, -356 in human and -23 in mouse) drives circadian expression of Per2 through Clock/Bmall-mediated transcriptional activation (Fig. 2a) (Yoo et al. 2005; Akashi et al. 2006). Interestingly, this regulation appears to be tissue-specific, because absence of Bmall in the liver leaves cyclic Per 2 expression unaffected, whereas absence of Bmall in other tissues abolishes this expression (McDearmon et al. 2006). Tissue-specific regulation of Per 2 is underscored by the finding that its expression in the SCN and the liver is differentially affected by nutritional factors (Iwanaga et al. 2005).

Another important regulatory element appears to be a DBP/E4BP4-binding element (D-box). It influences Per2 expression by a repressor-antiphasic-to-activator mechanism, which generates high-amplitude transcriptional activity (Ueda et al. 2005). The mouse Per2 promoter contains two D-boxes, one at -151 (A site) and the other at +197 (B site) (Fig. 2a) with E4BP4 acting predominantly at the B site as a repressor (Ohno et al. 2007b). To start transcription, the tightly packed DNA in the promoter region of Per 2 must be opened. Rhythms in histone $\mathrm{H} 3$ acetylation and RNA polymerase II binding were observed to be synchronous with the Per2 mRNA rhythms, indicating that histone acetylation might influence the expression of this gene (Etchegaray et al. 2003).

Acute induction of Per2 seems to be mediated by a number of signaling pathways converging on the binding of phosphorylated cAMP-responsive element (CRE)binding protein (CREB) on the CRE element present in the mouse Per 2 promoter at -1606 (Fig. 2a). However, this CRE element makes the Per 2 promoter much less responsive, in contrast to similar elements in the Per 1 promoter (Travnickova-Bendova et al. 2002). It appears 
a
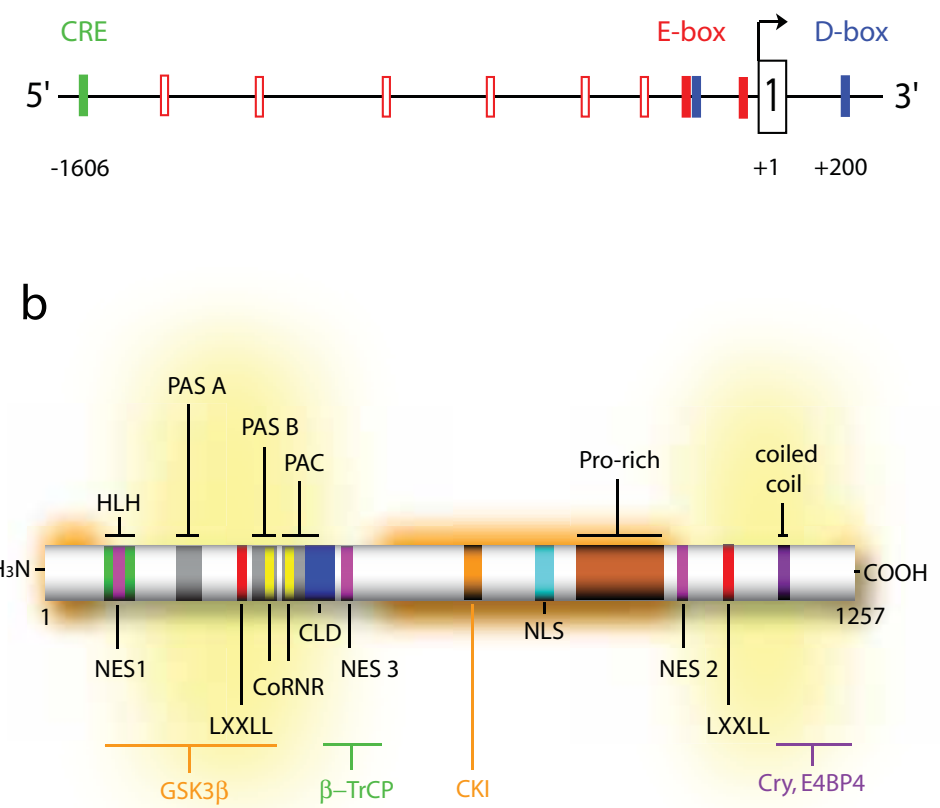

Figure 2. Murine Per2 promoter and structural domains of mouse PER2 protein. (a) Per2 promoter with CRE element (green), D boxes (blue), and E boxes (red). (Filled red box) E boxes sufficient for circadian expression of the gene; (open black box) exon 1; (arrow) transcription start site. Numbers below indicate the number of nucleotides upstream (negative values) or downstream (positive values) from the transcription start site $(+1)$. (b) Structural domains and functional motifs in the PER2 protein. The PAS domain consists of PAS A, PAS B, and the PAC motifs (gray). (HLH) Helix-loophelix motif (green); (NES1, 2, and 3) nuclear export sequence (pink); (LXXLL) motif found in coactivators to associate with nuclear receptors (red); (CoRNR) motif found in corepressors to associate with nuclear receptors (yellow); (CLD) cytoplasmic localization domain; (CKI) caseinkinase-binding site (orange); (NLS) nuclear localization sequence (cyan); (Pro-rich) prolinerich sequence (brown); (coiled-coil) dimerization domain (purple). (Yellow-shaded areas) Potential protein-binding domains; (orangeshaded areas) localization of phosphorylation sites. that in the mouse, Per1 acts as an adapter to entrain the circadian clock to changing light/dark cycles by regulating PER2 protein (Masubuchi et al. 2005). This is underscored by our finding that Per 2 can be induced in the late subjective night after a priming light pulse (Fig. 1c). Per1 or another factor, which must be induced first, is probably needed for acute Per 2 gene expression.

Taken together, the Per 2 promoter responds to circadian clock factors (CLOCK/BMAL1), factors providing feedback from peripheral clocks (DBP/E4BP4), and to a lesser extent, factors immediately inducing expression (pCREB). There are many more potential regulatory elements in the Per 2 promoter; however, their functional importance has to be established (Kornmann et al. 2007).

\section{STRUCTURAL DOMAINS AND FUNCTIONAL MOTIFS IN THE PER2 PROTEIN}

The PER2 protein contains several structural domains illustrated in Figure $2 \mathrm{~b}$. The mouse Per 2 gene product contains an amino-terminal helix-loop-helix (HLH) motif that is probably not binding to DNA, because the preceding amino acids are not basic. A large region termed the PAS (PER, ARNT, Sim) domain that contains two imperfect repeats (PAS A and PAS B) followed by a PAS-like PAC motif can be found carboxy-terminal to the HLH motif. The PAS domains in animals are implicated in protein-protein interaction and dimerization (Huang et al. 1993; Gekakis et al. 1995; Yildiz et al. 2005). Glycogen synthase kinase-3 $\beta$ (Gsk3- $\beta$ ) has been reported to interact with the PAS A and PAS B domains of PER2 (Iitaka et al. 2005). However, PAS/PAC domains are speculated to serve as ligand-binding domains (Ponting and Aravind 1997) that could bind heme and serve as oxygen sensors. Indeed, heme has been identified as a prosthetic group of
PER2 (Kaasik and Lee 2004), allowing the protein to sense redox status. Other evidence from bacteria suggests that PAS/PAC domains may act as light sensors resulting from FAD (flavin-adenine dinucleotide) binding (Hill et al. 1996; Bibikov et al. 1997). Although FAD binding has not been shown for PER2, it is known that Per 2 mutant mice, containing a PER2 protein lacking the carboxy-terminal half of the PAS B and the PAC domain (Zheng et al. 1999), show an altered behavioral response to light (Albrecht et al. 2001). Hence, it appears that the PAS/PAC domain in PER2 might enable this protein to sense cyclical variations in blue light and redox status.

Toward its carboxy-terminal end, the PER2 protein contains a coiled coil (Fig. 2b). This motif usually contains a repeated seven-amino-acid residue pattern forming a helix that can arrange with a helix of another protein to form a dimer that is held together by the burial of the hydrophobic surfaces in the helix dimer, hiding them from the water-filled environment. Interaction of the carboxy-terminal domain of PER2 with CRY (Miyazaki et al. 2001) and the leucine-zipper transcription factor E4BP4 has been reported (Ohno et al. 2007a). Hence, it appears that PER2 has the PAS domain plus a second structural feature that allows interaction with other proteins. Of note is that located between the PAS domain and the coiled-coil is a proline-rich sequence (Fig. 2b, brown). Proline residues make the amino acid chain less structured (no helices). Therefore, it appears that this prolinerich sequence divides the PER 2 protein into two structural entities that also might fold back for binding of one protein. If both domains interact with different proteins, PER2 could serve as a scaffold bringing proteins in proximity to each other to exert a specific function. This is actually not a new concept looking at how hormone receptors and their coactivators and corepressors work (McKenna et al. 1999; Stallcup et al. 2003). 
Interestingly, PER2 has some features found in coactivators and corepressors. For instance, LXXLL motifs (Fig. 2b, red) - where $\mathrm{L}$ is leucine and $X$ can be any amino acid - are found in both potential protein-protein interaction domains of PER2. This motif is found in different members of coactivators interacting with nuclear receptors as, for example, the steroid hormone receptor coactivator-1 (SRC-1) (Onate et al. 1995). SRC-1 interacts with different nuclear receptors including the progesterone receptor, estrogen receptor, PPAR, or RXR, stimulating their transcriptional potential. Similar activities could be postulated for PER2 function; however, no interaction between PER2 and a nuclear receptor has been reported.

Analogous to the situation with coactivators, corepressors contain two motifs related to the LXXLL sequences (Hu and Lazar 1999; Nagy et al. 1999; Perissi et al. 1999). These motifs, which exhibit a consensus sequence of $\mathrm{L} X X$ I/H I $X X X \mathrm{I} / \mathrm{L}$, have also been termed CoRNR boxes. Such CoRNR boxes are present in the PAS/PAC domain of PER2, and it remains to be seen whether they give PER2 the potential to act as corepressor.

\section{LOCALIZATION OF PER2 IN THE CELL}

Transfection studies using COS-7 and NIH-3T3 cells revealed that exogenously expressed PER2 is localized in the cytoplasm and in the nucleus (Yagita et al. 2002). Deletion analysis in PER2 showed that this protein contains functional nuclear import and export signals (CLD, NLS, and NES1-3 in Fig. 2b) (Yagita et al. 2000, 2002). Coexpression with CRY1 or CRY2 proteins promotes nuclear entry of PER2 (Kume et al. 1999; Yagita et al. 2000, 2002; Miyazaki et al. 2001) but CRY1 is not required for nuclear import of PER2. However, the interaction between PER2 and CRY proteins appears to be important to prevent ubiquitylation of both proteins prolonging their presence in the cell (Yagita et al. 2002). Our own studies investigating the localization of PER2 in the cell using enhanced green fluorescent protein (EGFP)tagged PER2 in nuclear escorting assays are largely in agreement with these previous studies (Fig. 3). We used human embryonic retinoblasts (HER) containing a partial adenovirus 5 (Ad5) genome. These cells are termed HER911 and have the advantage that they divide very slowly (about once in 40 hours; Fallaux et al. 1996) and therefore are very suitable to study localization of proteins over a longer period of time without cell division confounding the results. PER2-EGFP expressed in these cells is found in the nucleus, although some protein can be observed in the cytoplasm (Fig. 3). Other laboratories find PER2, when expressed alone, predominantly in the cytoplasm (Vielhaber et al. 2000). This discrepancy might be due to different cell lines used, indicating that modulation of the clock might be cell-type- and tissue-specific. Coexpression with CRY1 or CRY2 localizes PER2-EGFP entirely to the nucleus (Fig. 3, 2-4). Deletion of the CoRNR box in the PAS B/PAC domain leaves the protein largely in the cytoplasm, indicating that this motif has a role in nuclear escorting. Interestingly, CRY1 and CRY2 can transport this mutated form of PER2 to some extent to the nucleus (Fig. 3, 5-7). This indicates that either the CRY proteins interact at the CoRNR motif of PER2 for nuclear transport and/or that other proteins might influence nuclear translocation of PER2 through that motif. The deletion of the nuclear localization sequence (NLS) leaves the protein as expected in the cytoplasm. However, CRY1 and CRY2 can escort PER2 without NLS into the nucleus (Fig. 3, 9-11). Deletion of both the CoRNR motif and the NLS leaves the PER2 fusion protein largely in the cytoplasm, even when CRY proteins are coexpressed (Fig. 3, 12-14). These results suggest that the NLS in PER2 is not alone responsible for efficient nuclear localization, because deletion of the CoRNR motif in PER2 still containing the NLS leaves the protein in the cytoplasm. This indicates cooperativity between the NLS and CoRNR motifs with a possible involvement of CRY proteins. Similar results have been reported for PER3 (Yagita et al. 2000).

\section{PHOSPHORYLATION OF PER2}

Regulating the circadian clock through phosphorylation of its components appears to be a common theme in chronobiology (Edery et al. 1994; Tomita et al. 2005; Schafmeier et al. 2006). In PER2, more than 20 potential phosphorylation sites have been identified (Fig. 2b, orange shaded areas) (Schlosser et al. 2005; Vanselow et al. 2006) of which one site has received special attention in recent years. Patients with a specific form of familial advanced sleep phase syndrome have the mutation S662G in their PER2 protein, leading to a loss of binding of casein kinase $1 \varepsilon / \delta(\mathrm{CKI} \varepsilon / \delta)$ and hypophosphorylation of PER2 (Toh et al. 2001). This leads to a shortened period length of the circadian clock. In vitro studies and mouse genetics revealed that alteration of this site (662 in human, 659 in mice) from $\mathrm{S}$ to $\mathrm{G}$ recapitulates the finding in humans. Furthermore, a change from $\mathrm{S}$ to $\mathrm{D}$, mimicking constitutive phosphorylation charge and binding of CKI $\delta$, increased phosphorylation of PER2 leading to a longer period length (Fig. 4) (Vanselow et al. 2006; Xu et al. 2007). This mutation also increased the amount of PER2 protein observed in cells and tissues, correlating phosphorylation with protein abundance and period length (Fig. 4) (Vanselow et al. 2006; $\mathrm{Xu}$ et al. 2007). PER2 protein abundance seems to be regulated at least in vitro by $\beta$-TrCP $(\beta$-transducin repeat-containing protein), an ubiquitin adapter protein, that binds CKIE phosphorylated PER2. This leads to polyubiquitination of PER2 followed by proteasomemediated degradation (Eide et al. 2005).

In the future, it will be important to find the initiating kinase (red kinase in Fig. 4) that phosphorylates S662 (S659 in mice) and initiates the modulation of PER2 stability and variation of period length. A candidate could be Gsk-3 $\beta$, a homolog of the Drosophila gene shaggy known to have a role in clock modulation (Martinek et al. 2001) and PER2 phosphorylation (Iitaka et al. 2005). However, it has not been determined where Gsk- $3 \beta$ phosphorylates PER2. The studies mentioned above all highlight the importance of the phosphorylation status of PER2 to modulate the clock. To balance the amount of phosphorylation, phosphatases seem to be critical. Protein phosphatase 1 (PP1) has been shown to remove phosphates on PER2, leading to its accelerated ubiquitin and proteasome-mediated degradation (Gallego et al. 2006). Although PER2 has no characteristic RVXF motif to which PP1 binds directly, regulators of PP1, such as I-1 and Darpp-32, whose expression varies during the circa- 


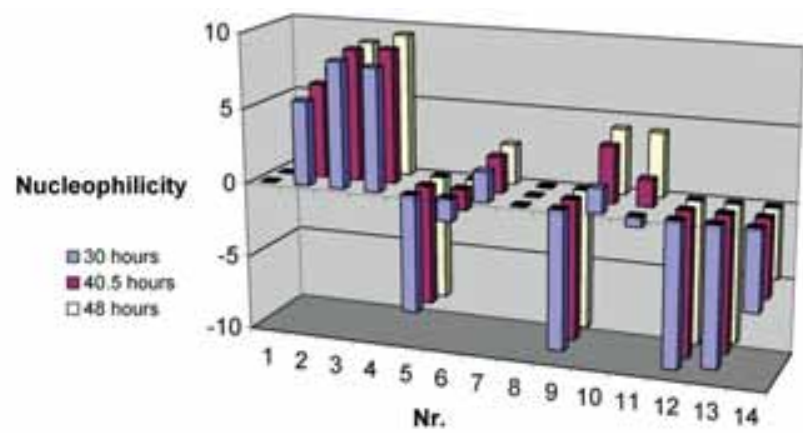

control
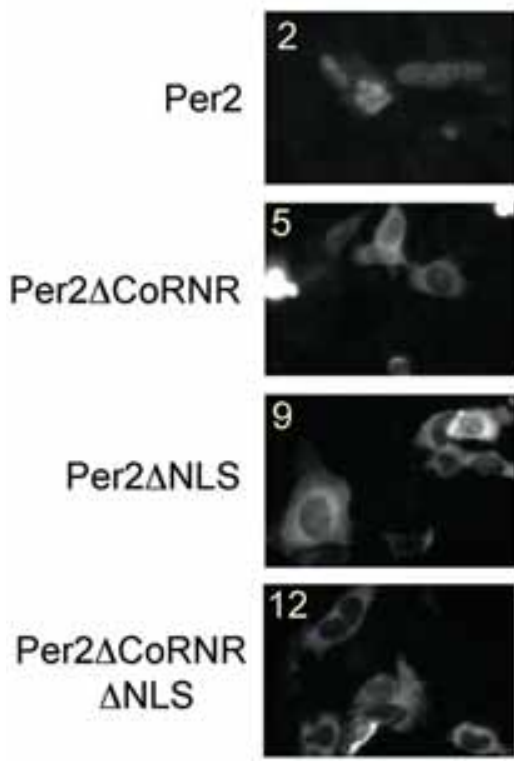

Cry1
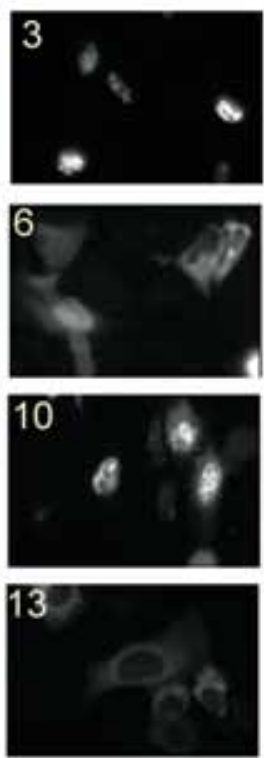

Cry2
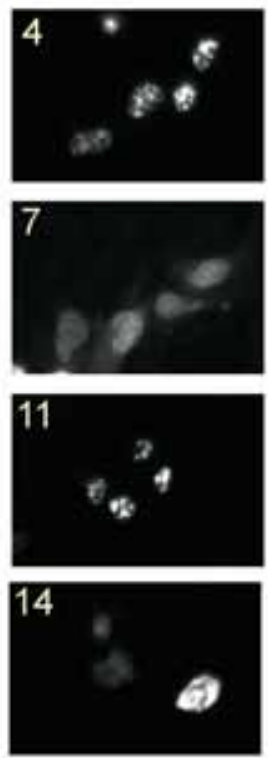

Figure 3. Nuclear escorting of PER2::GFP-fusion proteins. Human embryonic retinoblasts (HER911) were transfected with constructs coding for PER2::GFP or variants with deletions of the CoRNR and/or NLS motifs in PER2 (3 $\mu$ g each). pcDNA3.1 vector containing no insert (control), CRY1, or CRY2 was cotransfected ( $3 \mu \mathrm{g}$ each), and nucleophilicity of PER2::GFP-fusion proteins was assessed $30,40.5$, and 48 hours after transfection (upper panel). (Lower panel) Transfection scheme with typical illustrations 48 hours after transfection. (Light yellow numbers) Corresponding column in the summary graph in the upper panel. Columns 1 and 8 indicate the even distribution of the nonfused GFP protein in cells. Transfections were made with the linear polyethyleneimine method, and after fixation, the cells expressing GFP-tagged proteins were counted with the investigator not knowing the transfection scheme. The GFPfusion proteins were assigned to five different categories according to their nuclear versus cytosolic fluorescence. $\mathrm{N}>>\mathrm{C}$, $\mathrm{N}>\mathrm{C}$, $\mathrm{N}=\mathrm{C}, \mathrm{N}<\mathrm{C}, \mathrm{N}<<\mathrm{C}$ with $\mathrm{N}$ standing for nuclear and $\mathrm{C}$ for cytosolic localization. For every time point, the experiment was performed three times.

dian cycle (Ueda et al. 2002), might be capable of targeting PP1 to PER2 in a time-dependent fashion.

A recent study reports that posttranscriptional regulation, such as phosphorylation, is sufficient to generate oscillations in a clock protein of cyanobacteria (Nakajima et al. 2005). Whether this could be the case in mammals was studied by inserting a single copy of exogenous Per2 under the elongation factor- $1 \alpha(\mathrm{EF}-1 \alpha)$ promoter into NIH-3T3 fibroblasts, using a Flp-in system (Fujimoto et al. 2006). Interestingly, the exogenous PER2 protein oscillated without its coding mRNA cycling. Although the detailed mechanism regulating this process remains to be investigated, the bets are on phosphorylation and other posttranscriptional processes.

\section{INVOLVEMENT OF PER2 IN BIOLOGICAL FUNCTIONS}

\section{Circadian Rhythms}

The Per2 gene has been discovered in a search for molecular components that make up the circadian clock. Subsequently, mutation analysis revealed that this gene has a prominent role in circadian clock function (Zheng et al. 1999, 2001; Bae et al. 2001) because lack of a functional PER2 protein resulted in gradual loss of circadian rhythmicity in wheel-running behavior as well as in cellular clock function (Brown et al. 2005). Interestingly, Per2 affects the core clock mechanism by regulating Bmall expression in a positive manner (Shearman et al. 
a

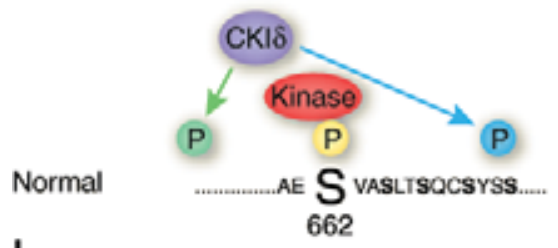

b

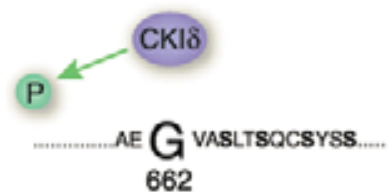

C

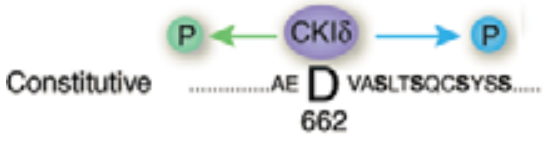

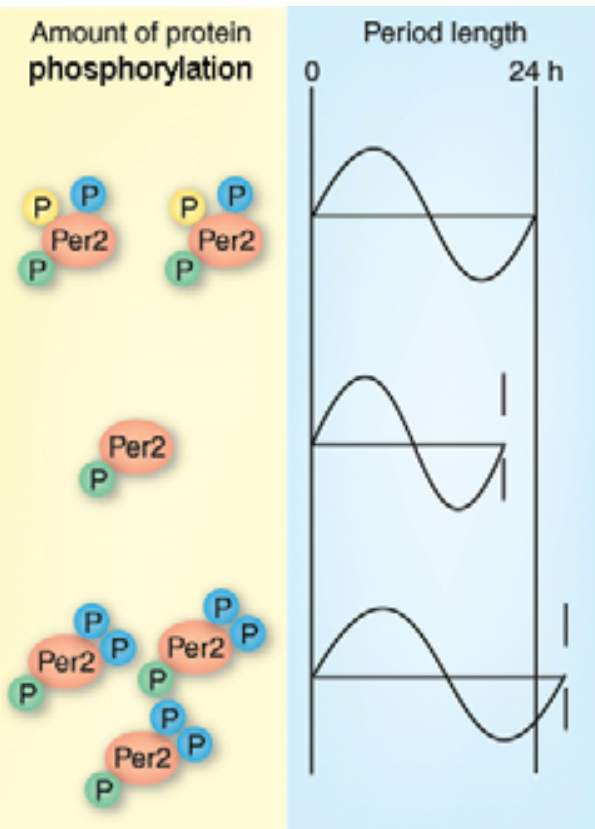

Figure 4. Differential phosphorylation of PER2 modulates period length of the circadian clock. (a) Amino acid sequence of normal human PER2 with significant serine (S) residues in bold. S662 is phosphorylated (yellow) by an unknown kinase (red), enabling casein kinase I $\delta(\mathrm{CKI} \delta$ ) to bind and phosphorylate adjacent serine residues (blue path). Independently of S662 phosphorylation, CKI $\delta$ phosphorylates other residues in PER2 (green path). This leads to intermediate levels of PER2 protein defining a period length of 24 hours. (b) Sequence of PER2 with the FASPS amino acid change S662G. CKI $\delta$ phosphorylates PER2 only through the green path. This leads to low levels of PER2 protein and a shortening of the clock period. (c) S662D mutation leads to constitutive binding of CKI $\delta$ and phosphorylation of adjacent S residues (blue path). Phosphorylation on other sites still occurs (green path). This leads to elevated levels of PER2 and a long-period length. (Reprinted, with permission, from Albrecht 2007 [CNature Publishing Group].)

2000; Oster et al. 2002). Astonishingly, deletion of Cry2 from Per 2 mutant mice reestablished circadian behavior (Oster et al. 2002). It remains to be seen how this is possible at the molecular level.

Because Per 2 is inducible by a light pulse in the early subjective night but not in the late subjective night (see Fig. 1a,b), it was speculated that it has a function in delaying clock phase. In agreement with that notion, Per2 mutant mice show impaired clock resetting, especially in the delay domain (Albrecht et al. 2001; Spoelstra et al. 2004). These findings indicate a function of Per 2 in clock resetting. Consistent with such a function is the finding that a mutation in the human Per2 gene, which abolishes normal phosphorylation of its protein, leads to familial advanced sleep phase syndrome (see above and Fig. 4b) (Toh et al. 2001). Mutation experiments in mice have confirmed that alteration of the PER2 phosphorylation pattern through mutation of the Per2 gene leads to clock period alterations (Vanselow et al. 2006; Xu et al. 2007). A single-nucleotide polymorphism in the $5^{\prime}$-untranslated region of the human Per2 gene has been associated with diurnal preference (Carpen et al. 2005), and a case study of a patient with recurrent hypersomnia revealed differences in Per 2 gene expression in remission and hypersomnia (Tomoda et al. 2003), further illustrating an involvement of this gene in clock adaptation and sleep regulation.

\section{Cancer}

Mice without functional PER2 are prone to develop cancer and display altered expression of genes involved in cell cycle regulation and tumor suppression such as cyclin D1, cyclin A, Myc, and Mdm2 (Fu et al. 2002). In particular, Myc is controlled directly by circadian regulators including Per2. Therefore, it appears that PER2 has a role in tumor suppression by regulating DNA-damage-responsive pathways. This notion is bolstered by the observation that expression of Per $1, \operatorname{Per} 2$, and Per 3 is deregulated in breast cancer tissue (Chen et al. 2005), although it is not clear whether this is the cause or the consequence of breast cancer. Improvement of tumor control in mice bearing Glasgow osteosarcoma was achieved by using seliciclib, a cyclin-dependent kinase inhibitor, which increased amplitudes of various clock genes including Per2 (Iurisci et al. 2006). Furthermore, overexpression of Per2 seems to induce cancer cell apoptosis (Hua et al. 2006), which will have to be confirmed by complementary studies. Interestingly, however, Per2 was identified in a large-scale RNA interference (RNAi) screen in human cells to be a component of the $\mathrm{p} 53$ pathway (Berns et al. 2004), strongly supporting a role of Per2 in tumor suppression. However, it is entirely unclear how PER 2 is involved in that process mechanistically.

\section{Immune System}

Recent evidence suggests an involvement of the Per2 gene in the immune system. Mice carrying a loss-of-function mutation of the Per2 gene display a loss of interferon$\gamma($ IFN- $\gamma$ ) mRNA cycling in the spleen (Arjona and Sarkar 2006). Furthermore, Per2-deficient mice are more resistant to lipopolysaccharide-induced endotoxic shock than wild- 
type mice. This is accompanied by decreased levels of proinflamatory cytokines such as IFN- $\gamma$ and interleukin- $1 \beta$ in the serum. The impaired IFN- $\gamma$ production seems to be attributable to defective natural killer cell function (Liu et al. 2006). This is an interesting observation in view of the involvement of Per2 in cancer development described above, because IFN- $\gamma$ activates macrophage and antitumor functions. How Per 2 impinges on the production of IFN- $\gamma$ and function of natural killer cells remains to be discovered.

\section{Cardiovascular System}

Disturbed diurnal rhythm alters Per2 gene expression and exacerbates cardiovascular disease in a mouse model of pressure overload cardiac hypertrophy. Upon resynchronization of the environmental light/dark cycle with the animal's internal cycle, cardiovascular parameters as well as Per 2 gene expression are normalized (Martino et al. 2007), suggesting a potential involvement of Per2 in the cardiovascular system. In agreement with this notion is the finding that mice with a loss-of-function mutation in PER2 display altered vascular endothelial function due to a decreased production of nitric oxide and vasodilatory prostaglandins as well as an increased release of COX-1derived vasoconstrictors in aortic rings (Viswambharan et al. 2007). Whether COX-1 is a direct target gene of PER2 and the clock mechanism or is regulated secondarily remains to be investigated. Interestingly, vascular endothelial growth factor (VEGF) appears to be regulated by clock components including PER2 as a negative regulator (Koyanagi et al. 2003). This finding is consistent with a role of PER2 as a tumor suppressor because it reduces expression of VEGF in hypoxic tumor cells, leading to reduction of vascularization of tumors.

\section{Metabolism}

That Per2 is involved in metabolic control is suggested by a study investigating the hepatic proteome in normal and Per 2 mutant mice (Reddy et al. 2006). In this study, the authors find that many proteins that cycle in their expression over 24 hours lose this property if Per 2 is absent (e.g., aldolase, arginase, and catalase). The alteration of arginase in these mice is consistent with the finding that these animals have abnormal endothelial function due to an alteration in NO (nitric oxide) signaling (see above) (Viswambharan et al. 2007), because arginase is a critical enzyme in NO metabolism. Furthermore, Per2 mutant mice show reduced endurance of muscles accompanied by increased levels of glycolytic enzymes in the anterior tibialis muscle, indicating a greater dependence on anaerobic metabolism under stress conditions (Bae et al. 2006). This is consistent with our own findings that Per 2 mutant mice show reduced muscle strength under stress conditions as revealed by the hanging wire test, in which the latency time for falling is much shorter for Per2 mice compared to wild-type animals (Fig. 5).

A recent study highlights the function of PER2 in connecting the clock and metabolism by showing that PER2 not only is a part of the circadian clock influencing signaling and metabolic pathways, but also responds to systemic cues, thereby linking the clock and metabolism in a interdependent fashion (Kornmann et al. 2007). PER2

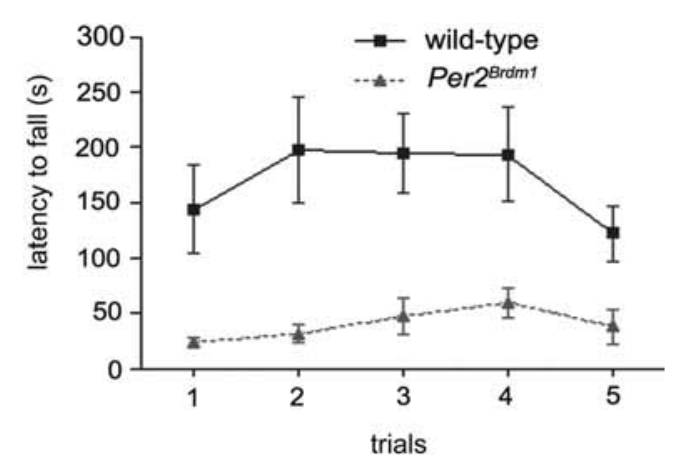

Figure 5. Muscle endurance in mice with and without functional PER2. Male mice (2-6 months old) were placed on a wire cage lid, which was turned upside down at a height of approximately $20 \mathrm{~cm}$ over a flat surface table. The time between the turning of the lid and the fall of the mouse was measured after the mice were familiar with this hanging-wire test. The latency to fall of wild-type (closed squares) and PER2 mutant (gray triangles) mice over five consecutive trials on 5 days was assessed. Twoway ANOVA with Bonferroni posthoc test revealed a significant difference for genotype with $p<0.001$ but no significant influence of time. Data points are represented as mean \pm S.E.M. with $n=6$ for each genotype.

appears to be the cogwheel that connects the liver clock with the organism's physiology and biochemistry.

\section{Nervous System}

Apart from its function in the clock mechanism in the SCN, Per2 appears to have additional functions in other brain areas. It is postulated that a food entrainable oscillator (FEO) resides in the brain, which is responsible for anticipatory activity in expectation of regularly scheduled meals. A mutation in Per2 leads to loss of food anticipatory activity in mice, suggesting an important role of Per2 in the FEO (Feillet et al. 2006). Because the mutation affects the whole organism, it remains to be shown that the observed loss-of-food anticipation in these mice is specific for Per 2 function in the brain and is not due to the role of Per2 in the liver.

Interestingly, several studies have found that overeating shares neurobiological mechanisms with the addictive properties of drugs of abuse (for review, see Simerly 2006). Because PER2 not only affects food anticipatory behavior, but also modulates the effects of drugs of abuse (Abarca et al. 2002; Spanagel et al. 2005), we postulate that PER2 influences the neurobiological circuitry that is common to feeding signals and drugs. Because specific areas of the midbrain such as the arcuate nucleus and the ventral tegmental area are integrating these signals, a specific function of PER2 in these brain regions can be envisaged.

That PER2 has a central role in sleep, especially in familial advanced sleep phase syndrome, has been described above. Because sleep appears to involve remodeling of neuronal connectivity by strengthening or weakening synaptic connections, it is conceivable to suspect an involvement of PER2 in these processes. A recent study shows that PER2 is involved in gating light/dark information to vesicular glutamate transporter 1 (vGLUT1) content on synaptic vesicles (Yelamanchili et al. 2006). This leads to alterations in glutamate filling of these vesicles. 


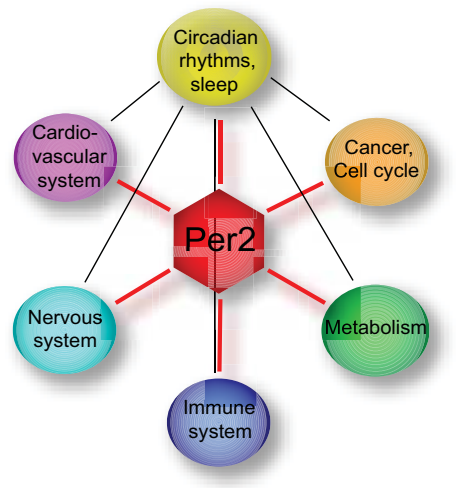

Figure 6. Schematic diagram illustrating various body functions influenced by PER2 (red lines) and other clock components (black lines)

Changing the amount of glutamate that can be released by neurons will impinge on synaptic strength. Hence, environmental signals such as light can have an impact on brain function. Therefore, one could assume that health problems in industrialized societies, where the natural day/night regime is largely ignored, are rooted in the misalignment between different clocks affecting normal brain function. As a consequence depression (Partonen et al. 2007), excessive alcohol consumption and overeating is observed.

\section{CONCLUSIONS}

In the past decade, many of the molecular components of the circadian clock have been identified in several organisms. The concept emerged that there is not only one clock ticking in the brain but virtually any tissue and every cell contains the cogwheels for a clock. How do these different clocks interact with each other to produce a coherent systemic rhythm useful for the entire organism? From the above description of the structural organization and function of PER2, it seems that this molecule unifies several characteristics necessary for a function to serve as an integrator (Fig. 6). Its promoter seems to be responsive to a plethora of transcriptional activators, the protein can be finely tuned in its stability and localization by posttranscriptional modifications, and it seems to interact with various proteins to mediate specific functions. Therefore, the next steps to understand the circadian system as a whole will be to study tissue-specific regulation of clock components and in particular PER2 function. Genetic tools to achieve this goal are available. At the protein level, resolving the protein structure will help to identify interacting partners, and potential pharmacological agents binding to PER2 might be discovered. This will help to find tissue-specific cooperation partners of this protein and provide explanations for the observed activator and repressor potential of PER2, elucidating the mysteries of this Janus-faced molecule.

\section{ACKNOWLEDGMENTS}

Support from the Swiss National Science Foundation, the Velux Foundation, and the European Union Project EUCLOCK is acknowledged.

\section{REFERENCES}

Abarca C., Albrecht U., and Spanagel R. 2002. Cocaine sensitization and reward are under the influence of circadian genes and rhythm. Proc. Natl. Acad. Sci. 99: 9026.

Akashi M., Ichise T., Mamine T., and Takumi T. 2006. Molecular mechanism of cell-autonomous circadian gene expression of Period2, a crucial regulator of the mammalian circadian clock. Mol. Biol. Cell 17: 555.

Albrecht U. 2007. Per2 has time on its side. Nat. Chem. Biol. 3: 139.

Albrecht U., Sun Z.S., Eichele G., and Lee C.C. 1997. A differential response of two putative mammalian circadian regulators, mper1 and mper2, to light. Cell 91: 1055.

Albrecht U., Zheng B., Larkin D., Sun Z.S., and Lee C.C. 2001. mPer 1 and mPer 2 are essential components for normal resetting of the circadian clock. J. Biol. Rhythms 16: 100.

Arjona A. and Sarkar D.K. 2006. The circadian gene mPer 2 regulates the daily rhythm of IFN-gamma. J. Interferon Cytokine Res. 26: 645.

Bae K., Jin X., Maywood E.S., Hastings M.H., Reppert S.M., and Weaver D. 2001. Differential functions of mPer 1, mPer2, and $m P e r 3$ in the SCN circadian clock. Neuron 30: 525.

Bae K., Lee K., Seo Y., Lee H., Kim D., and Choi I. 2006. Differential effects of two period genes on the physiology and proteomic profiles of mouse anterior tibialis muscles. Mol. Cells 22: 275.

Berns K., Hijmans E.M., Mullenders J., Brummelkamp T.R., Velds A., Heimerikx M., Kerkhoven R.M., Madiredjo M., Nijkamp W., Weigelt B., Agami R., Ge W., Cavet G., Linsley P.S., Beijersbergen R.L., and Bernards R. 2004. A large-scale RNAi screen in human cells identifies new components of the p53 pathway. Nature 428: 431.

Bibikov S.I., Biran R., Rudd K.E., and Parkinson J.S. 1997. A signal transducer for aerotaxis in Escherichia coli. J. Bacteriol. 179: 4075.

Brown S.A., Fleury-Olela F., Nagoshi E., Hauser C., Juge C., Meier C.A., Chicheportiche R., Dayer J.M., Albrecht U., and Schibler U. 2005. The period length of fibroblast circadian gene expression varies widely among human individuals. PLoS Biol. 3: e338.

Cajochen C., Jud C., Munch M., Kobialka S., Wirz-Justice A., and Albrecht U. 2006. Evening exposure to blue light stimulates the expression of the clock gene PER2 in humans. Eur. J. Neurosci. 23: 1082.

Carpen J.D., Archer S.N., Skene D.J., Smits M., and von Schantz M. 2005. A single-nucleotide polymorphism in the $5^{\prime}$ untranslated region of the hPER2 gene is associated with diurnal preference. J. Sleep Res. 14: 293.

Chen S.T., Choo K.B., Hou M.F., Yeh K.T., Kuo S.J., and Chang J.G. 2005. Deregulated expression of the PER1, PER2 and PER3 genes in breast cancers. Carcinogenesis 26: 1241.

Edery I., Zwiebel L.J., Dembinska M.E., and Rosbash M. 1994. Temporal phosphorylation of the Drosophila period protein. Proc. Natl. Acad. Sci. 91: 2260.

Eide E.J., Woolf M.F., Kang H., Woolf P., Hurst W., Camacho F. Vielhaber E.L., Giovanni A., and Virshup D.M. 2005. Control of mammalian circadian rhythm by CKIE-regulated proteasome-mediated PER2 degradation. Mol. Cell. Biol. 25: 2795.

Etchegaray J.P., Lee C., Wade P.A., and Reppert S.M. 2003. Rhythmic histone acetylation underlies transcription in the mammalian circadian clock. Nature 421: 177.

Fallaux F.J., Kranenburg O., Cramer S.J., Houweling A., Van Ormondt H., Hoeben R.C., and Van Der Eb A.J. 1996. Characterization of 911: A new helper cell line for the titration and propagation of early region 1-deleted adenoviral vectors. Hum. Gene Ther. 7: 215.

Feillet C.A., Ripperger J.A., Magnone M.C., Dulloo A., Albrecht U., and Challet E. 2006. Lack of food anticipation in Per2 mutant mice. Curr. Biol. 16: 2016.

Fu L., Pelicano H., Liu J., Huang P., and Lee C.C. 2002. The circadian gene period 2 plays an important role in tumor suppression and DNA damage response in vivo. Cell 111: 41.

Fujimoto Y., Yagita K., and Okamura H. 2006. Does mPER2 protein oscillate without its coding mRNA cycling?: Posttranscriptional regulation by cell clock. Genes Cells 11: 525. Fukuhara C., Brewer J.M., Dirden J.C., Bittman E.L., Tosini G., 
and Harrington M.E. 2001. Neuropeptide Y rapidly reduces Period 1 and Period 2 mRNA levels in the hamster suprachiasmatic nucleus. Neurosci. Lett. 314: 119.

Gallego M., Kang H., and Virshup D.M. 2006. Protein phosphatase 1 regulates the stability of the circadian protein PER2. Biochem. J. 399: 169.

Gekakis N., Saez L., Delahaye-Brown A.M., Myers M.P., Sehgal A., Young M.W., and Weitz C.J. 1995. Isolation of timeless by PER protein interaction: Defective interaction between timeless protein and long-period mutant PERL. Science 270: 811.

Hill S., Austin S., Eydmann T., Jones T., and Dixon R. 1996. Azotobacter vinelandii NIFL is a flavoprotein that modulates transcriptional activation of nitrogen-fixation genes via a redox-sensitive switch. Proc. Natl. Acad. Sci. 93: 2143.

Horikawa K., Yokota S., Fuji K., Akiyama M., Moriya T., Okamura H., and Shibata S. 2000. Nonphotic entrainment by 5-HT1A/7 receptor agonists accompanied by reduced Per1 and Per2 mRNA levels in the suprachiasmatic nuclei. $J$. Neurosci. 20: 5867.

Hu X. and Lazar M.A. 1999. The CoRNR motif controls the recruitment of corepressors by nuclear hormone receptors. Nature 402: 93.

Hua H., Wang Y., Wan C., Liu Y., Zhu B., Yang C., Wang X., Wang Z., Cornelissen-Guillaume G., and Halberg F. 2006. Circadian gene mPer2 overexpression induces cancer cell apoptosis. Cancer Sci. 97: 589.

Huang Z.J., Edery I., and Rosbash M. 1993. PAS is a dimerization domain common to Drosophila period and several transcription factors. Nature 364: 259

Iitaka C., Miyazaki K., Akaike T., and Ishida N. 2005. A role for glycogen synthase kinase-3beta in the mammalian circadian clock. J. Biol. Chem. 280: 29397.

Iurisci I., Filipski E., Reinhardt J., Bach S., Gianella-Borradori A., Iacobelli S., Meijer L., and Levi F. 2006. Improved tumor control through circadian clock induction by Seliciclib, a cyclin-dependent kinase inhibitor. Cancer Res. 66: 10720.

Iwanaga H., Yano M., Miki H., Okada K., Azama T., Takiguchi S., Fujiwara Y., Yasuda T., Nakayama M., Kobayashi M., Oishi K., Ishida N., Nagai K., and Monden M. 2005. Per2 gene expressions in the suprachiasmatic nucleus and liver differentially respond to nutrition factors in rats. JPEN $J$. Parenter. Enteral Nutr. 29: 157.

Kaasik K. and Lee C.C. 2004. Reciprocal regulation of haem biosynthesis and the circadian clock in mammals. Nature $\mathbf{4 3 0}$ 467.

Kornmann B., Schaad O., Bujard H., Takahashi J.S., and Schibler U. 2007. System-driven and oscillator-dependent circadian transcription in mice with a conditionally active liver clock. PLoS Biol. 5: e34.

Koyanagi S., Kuramoto Y., Nakagawa H., Aramaki H., Ohdo S., Soeda S., and Shimeno H. 2003. A molecular mechanism regulating circadian expression of vascular endothelial growth factor in tumor cells. Cancer Res. 63: 7277.

Kume K., Zylka M.J., Sriram S., Shearman L.P., Weaver D.R., Jin X., Maywood E.S., Hastings M.H., and Reppert S.M. 1999. mCRY1 and mCRY2 are essential components of the negative limb of the circadian clock feedback loop. Cell $\mathbf{9 8 :}$ 193.

Liu J., Mankani G., Shi X., Meyer M., Cunningham-Runddles S., Ma X., and Sun Z.S. 2006. The circadian clock Period 2 gene regulates gamma interferon production of NK cells in host response to lipopolysaccharide-induced endotoxic shock. Infect. Immun. 74: 4750.

Martinek S., Inonog S., Manoukian A.S., and Young M.W. 2001. A role for the segment polarity gene shaggy/GSK-3 in the Drosophila circadian clock. Cell 105: 769.

Martino T.A., Tata N., Belsham D.D., Chalmers J., Straume M., Lee P., Pribiag H., Khaper N., Liu P.P., Dawood F., Backx P.H., Ralph M.R., and Sole M.J. 2007. Disturbed diurnal rhythm alters gene expression and exacerbates cardiovascular disease with rescue by resynchronization. Hypertension 49: 1104

Masubuchi S., Kataoka N., Sassone-Corsi P., and Okamura H. 2005. Mouse Period1 (mPER1) acts as a circadian adaptor to entrain the oscillator to environmental light/dark cycles by regulating mPER2 protein. J. Neurosci. 25: 4719.
McDearmon E.L., Patel K.N., Ko C.H., Walisser J.A., Schook A.C., Chong J.L., Wilsbacher L.D., Song E.J., Hong H.K., Bradfield C.A., and Takahashi J.S. 2006. Dissecting the functions of the mammalian clock protein BMAL1 by tissue-specific rescue in mice. Science 314: 1304.

McKenna N.J., Lanz R.B., and O’Malley B.W. 1999. Nuclear receptor coregulators: Cellular and molecular biology. Endocr. Rev. 20: 321

Miyazaki K., Mesaki M., and Ishida N. 2001. Nuclear entry mechanism of rat PER2 (rPER2): Role of rPER2 in nuclear localization of CRY protein. Mol. Cell. Biol. 21: 6651.

Nagase T., Ishikawa K., Nakajima D., Ohira M., Seki N., Miyajima N., Tanaka A., Kotani H., Nomura N., and Ohara O. 1997. Prediction of the coding sequences of unidentified human genes. VII. The complete sequences of 100 new cDNA clones from brain which can code for large proteins in vitro. DNA Res. 4: 141.

Nagy L., Kao H.Y., Love J.D., Li C., Banayo E., Gooch J.T., Krishna V., Chatterjee K., Evans R.M., and Schwabe J.W. 1999. Mechanism of corepressor binding and release from nuclear hormone receptors. Genes Dev. 13: 3209.

Nakajima M., Imai K., Ito H., Nishiwaki T., Murayama Y., Iwasaki H., Oyama T., and Kondo T. 2005. Reconstitution of circadian oscillation of cyanobacterial KaiC phosphorylation in vitro. Science 308: 414.

Nielsen H.S., Hannibal J., and Fahrenkrug J. 2002. Vasoactive intestinal polypeptide induces per1 and per2 gene expression in the rat suprachiasmatic nucleus late at night. Eur. $J$. Neurosci. 15: 570.

Ohno T., Onishi Y., and Ishida N. 2007a. The negative transcription factor E4BP4 is associated with circadian clock protein PERIOD2. Biochem. Biophys. Res. Commun. 354: 1010. . 2007b. A novel E4BP4 element drives circadian expression of mPeriod2. Nucleic Acids Res. 35: 648.

Onate S.A., Tsai S.Y., Tsai M.J., and O'Malley B.W. 1995. Sequence and characterization of a coactivator for the steroid hormone receptor superfamily. Science 270: 1354.

Oster H., Yasui A., van der Horst G.T., and Albrecht U. 2002. Disruption of mCry2 restores circadian rhythmicity in mPer2 mutant mice. Genes Dev. 16: 2633.

Partonen T., Treutlein J., Alpman A., Frank J., Johansson C., Depner M., Aron L., Rietschel M., Wellek S., Soronen P., Paunio T., Koch A., Chen P., Lathrop M., Adolfsson R., Persson M.L., Kasper S., Schalling M., Peltonen L., and Schumann G. 2007. Three circadian clock genes Per2, Arntl, and Npas2 contribute to winter depression. Ann. Med. 39: 229.

Perissi V., Staszewski L.M., McInerney E.M., Kurokawa R., Krones A., Rose D.W., Lambert M.H., Milburn M.V., Glass C.K., and Rosenfeld M.G. 1999. Molecular determinants of nuclear receptor-corepressor interaction. Genes Dev. 13: 3198.

Ponting C.P. and Aravind L. 1997. PAS: A multifunctional domain family comes to light. Curr. Biol. 7: R674.

Reddy A.B., Karp N.A., Maywood E.S., Sage E.A., Deery M., O’Neill J.S., Wong G.K., Chesham J., Odell M., Lilley K.S., Kyriacou C.P., and Hastings M.H. 2006. Circadian orchestration of the hepatic proteome. Curr. Biol. 16: 1107.

Schafmeier T., Kaldi K., Diernfellner A., Mohr C., and Brunner M. 2006. Phosphorylation-dependent maturation of Neurospora circadian clock protein from a nuclear repressor toward a cytoplasmic activator. Genes Dev. 20: 297.

Schlosser A., Vanselow J.T., and Kramer A. 2005. Mapping of phosphorylation sites by a multi-protease approach with specific phosphopeptide enrichment and NanoLC-MS/MS analysis. Anal. Chem. 77: 5243

Segall L.A., Perrin J.S., Walker C.D., Stewart J., and Amir S. 2006. Glucocorticoid rhythms control the rhythm of expression of the clock protein, Period2, in oval nucleus of the bed nucleus of the stria terminalis and central nucleus of the amygdala in rats. Neuroscience 140: 753 .

Shearman L.P., Zylka M.J., Weaver D.R., Kolakowski L.F., and Reppert S.M. 1997. Two period homologs: Circadian expression and photic regulation in the suprachiasmatic nuclei. Neuron 19: 1261 .

Shearman L.P., Sriram S., Weaver D.R., Maywood E.S., Chaves I., Zheng B., Kume K., Lee C.C., van der Horst G.T., Hastings 
M.H., and Reppert S.M. 2000. Interacting molecular loops in the mammalian circadian clock. Science 288: 1013.

Simerly R. 2006. Feeding signals and drugs meet in the midbrain. Nat. Med. 12: 1244 .

Spanagel R., Pendyala G., Abarca C., Zghoul T., Sanchis-Segura C., Magnone M.C., Lascorz J., Depner M., Holzberg D., Soyka M., Schreiber S., Matsuda F., Lathrop M., Schumann G., and Albrecht U. 2005. The clock gene Per2 influences the glutamatergic system and modulates alcohol consumption. Nat. Med. 11: 35.

Spoelstra K., Albrecht U., van der Horst G.T., Brauer V., and Daan S. 2004. Phase responses to light pulses in mice lacking functional per or cry genes. J. Biol. Rhythms 19: 518 .

Stallcup M.R., Kim J.H., Teyssier C., Lee Y.H., Ma H., and Chen D. 2003. The roles of protein-protein interactions and protein methylation in transcriptional activation by nuclear receptors and their coactivators. J. Steroid Biochem. Mol. Biol. 85: 139 .

Toh K.L., Jones C.R., He Y., Eide E.J., Hinz W.A., Virshup D.M., Ptacek L.J., and Fu Y.-H. 2001. An hPer2 phosphorylation site mutation in familial advanced sleep phase syndrome. Science 291: 1040.

Tomita J., Nakajima M., Kondo T., and Iwasaki H. 2005. No transcription-translation feedback in circadian rhythm of KaiC phosphorylation. Science 307: 251.

Tomoda A., Joudoi T., Kawatani J., Ohmura T., Hamada A., Tonooka S., and Miike T. 2003. Case study: Differences in human Per2 gene expression, body temperature, cortisol, and melatonin parameters in remission and hypersomnia in a patient with recurrent hypersomnia. Chronobiol. Int 20: 893.

Travnickova-Bendova Z., Cermakian N., Reppert S.M., and Sassone-Corsi P. 2002. Bimodal regulation of mPeriod promoters by CREB-dependent signaling and CLOCK/BMAL1 activity. Proc. Natl. Acad. Sci. 99: 7728.

Ueda H.R., Hayashi S., Chen W., Sano M., Machida M., Shigeyoshi Y., Iino M., and Hashimoto S. 2005. System-level identification of transcriptional circuits underlying mammalian circadian clocks. Nat. Genet. 37: 187.

Ueda H.R., Chen W., Adachi A., Wakamatsu H., Hayashi S., Takasugi T., Nagano M., Nakahama K., Suzuki Y., Sugano S., Iino M., Shigeyoshi Y., and Hashimoto S. 2002. A transcription factor response element for gene expression during circadian night. Nature 418: 534.

Vanselow K., Vanselow J.T., Westermark P.O., Reischl S., Maier B., Korte T., Herrmann A., Herzel H., Schlosser A., and Kramer A. 2006. Differential effects of PER2 phosphorylation: Molecular basis for the human familial advanced sleep phase syndrome (FASPS). Genes Dev. 20: 2660.

Vielhaber E., Eide E., Rivers A., Gao Z.H., and Virshup D.M.
2000. Nuclear entry of the circadian regulator mPER 1 is controlled by mammalian casein kinase I epsilon. Mol. Cell. Biol. 20: 4888

Viswambharan H., Carvas J.M., Antic V., Marecic A., Jud C., Zaugg C.E., Ming X.F., Montani J.P., Albrecht U., and Yang Z. 2007. Mutation of the circadian clock gene Per2 alters vascular endothelial function. Circulation 115: 2188

von Schantz M., Jenkins A., and Archer S.N. 2006. Evolutionary history of the vertebrate period genes. J. Mol. Evol. 62: 701 .

Wijnen H. and Young M.W. 2006. Interplay of circadian clocks and metabolic rhythms. Annu. Rev. Genet. 40: 409.

Xu Y., Toh K.L., Jones C.R., Shin J.Y., Fu Y.H., and Ptacek L.J. 2007. Modeling of a human circadian mutation yields insights into clock regulation by PER2. Cell 128: 59 .

Yagita K., Tamanini F., Yasuda M., Hoeijmakers J.H.J., van der Horst G.T.J., and Okamura H. 2002. Nucleocytoplasmic shuttling and $\mathrm{mCRY}$-dependent inhibition of ubiquitylation of the mPER2 clock protein. EMBO J. 21: 1301.

Yagita K., Yamaguchi S., Tamanini F., van der Horst G.T.J., Hoeijmakers J.H.J., Yasui A., Loros J.J., Dunlap J.C., and Okamura H. 2000. Dimerization and nuclear entry of mPER proteins in mammalian cells. Genes Dev. 14: 1353.

Yan L. and Silver R. 2002. Differential induction and localization of mPer1 and mPer2 during advancing and delaying phase shifts. Eur. J. Neurosci. 16: 1531.

Yannielli P.C., Molyneux P.C., Harrington M.E., and Golombek D.A. 2007. Ghrelin effects on the circadian system of mice. $J$. Neurosci. 27: 2890.

Yelamanchili S.V., Pendyala G., Brunk I., Darna M., Albrecht U., and Ahnert-Hilger G. 2006. Differential sorting of the vesicular glutamate transporter 1 into a defined vesicular pool is regulated by light signaling involving the clock gene Period2. J. Biol. Chem. 281: 15671.

Yildiz O., Doi M., Yujnovsky I., Cardone L., Berndt A., Hennig S., Schulze S., Urbanke C., Sassone-Corsi P., and Wolf E. 2005. Crystal structure and interactions of the PAS repeat region of the Drosophila clock protein PERIOD. Mol. Cell 17: 69.

Yoo S.H., Ko C.H., Lowrey P.L., Buhr E.D., Song E.J., Chang S., Yoo O.J., Yamazaki S., Lee C., and Takahashi J.S. 2005. A noncanonical E-box enhancer drives mouse Period2 circadian oscillations in vivo. Proc. Natl. Acad. Sci. 102: 2608.

Zheng B., Larkin D.W., Albrecht U., Sun Z.S., Sage M., Eichele G., Lee C.C., and Bradley A. 1999. The $m$ Per2 gene encodes a functional component of the mammalian circadian clock. Nature 400: 169

Zheng B., Albrecht U., Kaasik K., Sage M., Lu W., Vaishnav S. Li Q., Sun Z.S., Eichele G., Bradley A., and Lee C.C. 2001. Nonredundant roles of the mPer1 and mPer2 genes in the mammalian circadian clock. Cell 105: 683. 


\section{$8_{\mathrm{CSH}}^{\infty} \mathrm{CH}$ Cold Spring Harbor Symposia SYMPOSIA}

\section{The Multiple Facets of Per2}

U. Albrecht, A. Bordon, I. Schmutz, et al.

Cold Spring Harb Symp Quant Biol 2007 72: 95-104

Access the most recent version at doi:10.1101/sqb.2007.72.001

References This article cites 85 articles, 35 of which can be accessed free at: http://symposium.cshlp.org/content/72/95.full.html\#ref-list-1

\section{License} Email Alerting $\begin{aligned} & \text { Receive free email alerts when new articles cite this article - sign up in the box at the } \\ & \text { Service }\end{aligned}$ top right corner of the article or click here. 